\title{
Factors associated with survival in patients with meningioma
}

\section{Bridget J. McCarthy, Ph.D., Faith Davis, Ph.D., Sally Freels, Ph.D., Tanya S. Surawicz, M.P.H., Denise Damek, M.D., James Grutsch, Ph.D., Herman R. Menck, M.B.A., and Edward R. Laws, Jr., M.D.}

Department of Biostatistics/Epidemiology, University of Illinois at Chicago, Chicago, Illinois; Department of Neuro-Oncology/Neurology, Massachusetts General Hospital and Harvard Medical School, Boston, Massachusetts; Division of Research, Chicago Institute of Neurosurgery and Neuroscience, Chicago, Illinois; National Cancer Data Base Commission, American College of Surgeons, Chicago, Illinois; and Department of Neurological Surgery, University of Virginia Health Sciences Center, Charlottesville, Virginia

Factors affecting the survival rate in patients with meningiomas were explored using the National Cancer Database (NCDB), which includes tumors from approximately 1000 hospitals participating in the American College of Surgeons tumor registry program. Analysis included over 9000 cases diagnosed from 1985 to 1988 and 1990 to 1992. Survival estimates were computed and prognostic factors were identified using a proportional hazards model. The overall 5-year survival rate was $69 \%$ and it declined with age. This rate was $81 \%$ in patients aged 21 to 64 and $56 \%$ for patients 65 years of age or older. When patients were grouped by the histological type of their tumors, those with benign tumors had an overall 5-year survival rate of 70\%, whereas the overall 5-year survival rates in patients with atypical and malignant meningiomas were $75 \%$ and $55 \%$, respectively. Prognostic factors for benign tumors included age at diagnosis, tumor size, whether treated surgically, hospital type, and radiation therapy; for malignant tumors, age at diagnosis, whether treated surgically, and radiation therapy were statistically significant. The 5-year rate for recurrence of symptoms (regardless of the method of treatment) was $18.2 \%$ for those with benign tumors and $27.5 \%$ for those with malignant tumors. In patients whose benign tumor had been completely removed, the 5-year rate of tumor recurrence was $20.5 \%$. Although not population-based, the NCDB has the potential for providing pertinent information regarding patient characteristics and methods of treatment for benign, as well as malignant, brain tumors.

\section{Key Words * meningioma * survival * prognosis}

A recent population-based study in Australia reported a 5-year survival rate for patients harboring meningioma which was lower in men than in women.[14] In a previous analysis based on data from the National Cancer Database (NCDB) of the American College of Surgeons and the American Cancer Society, a gender difference in survival rates was also noted for meningioma, but it was not apparent for other types of brain tumors.[19] These results were surprising, because a carefully documented clinical series has not identified gender as an independent prognostic factor with respect to this tumor.[6] It has been suggested that these gender differences may reflect a larger proportion of malignant disease in men 
than in women (Susan Preston-Martin, personal communication, 1996). Conversely, other prognostic factors, more closely related to treatment or pathogenesis of the disease, may explain the lack of gender differences in clinical series in the survival of patients with meningiomas.

The current study uses a very large hospital-based, voluntary sample of patients with both benign and malignant brain tumors, with the first two calls for data representing approximately $39 \%$ of all brain tumors in the United States.[11] Although this database is not population based, it is one of the few in the United States that collects information on benign tumors. Registries such as Surveillance, Epidemiology, and End Results, although population based, only collect information on malignant tumors and cannot adequately assess survival in patients with meningiomas, which are primarily benign. The objective of this study was to evaluate differences in prognostic factors impacting the survival rates of patients with meningiomas, from whom data were stored in the NCDB. Separate models for benign and malignant tumors were used. Factors influencing time to recurrence of symptoms and functional capability were also explored.

\section{CLINICAL MATERIAL AND METHODS}

\section{Patient Selection}

Data on individuals with brain and central nervous system tumors were obtained from the NCDB, a nonrandom voluntary sample of cancer cases in the United States compiled by the Commission on Cancer of the American College of Surgeons and the American Cancer Society.[10,11] Information on patients with primary and malignant brain tumors from approximately 1000 of the 2200 hospitals accredited by the Commission on Cancer for the years 1985 to 1988 and 1990 to 1992 was obtained voluntarily for the NCDB. Data for the year 1989 were not collected.

\section{Study Methodology}

Cancer cases were registered by specially trained registrars who recorded information on demographic characteristics such as gender, race, and age at diagnosis, and clinical prognostic factors such as extent of surgery, type of radiation treatment, and tumor size and location. This information was collected in standardized computer-readable form from each of the institutions submitting data.[10] Each month a $10 \%$ sample of the abstracted patient data for each institution was reviewed by a member of the hospital cancer committee. A quality control assessment of the NCDB has been published previously.[18]

The duplicate records of patients seen at multiple institutions or with multiple surgical procedures were excluded from the data set. Those records that contained invalid codes for gender, date of diagnosis, vital status, date of last contact, or date of birth were also excluded from the analyses. International Classification of Diseases for Oncology (ICDO) codes 9530 to 9537 were used to select 9827 cases of meningioma from the larger data set.[20] Thirteen papillary meningiomas (ICDO 9538/1) and three meningeal sarcomatoses (ICDO 9539/3) were excluded from the data set. There was no case of an asymptomatic meningioma diagnosed at autopsy in the current study. Of these cases, $96.4 \%$ of benign and $97.2 \%$ of malignant meningiomas were treated at the reporting hospital.

\section{Tumor Classification}

Because of differences in predicted mortality rates, meningiomas were divided into three broad histological groupings. Benign meningiomas (8891) were defined by ICDO codes 9530/0 (meningioma, NOS); code 9530/1 (meningiomatosis, NOS/diffuse/multiple); code 9531 
(meningotheliomatous/endotheliomatous/syncytial); code 9532 (fibrous/fibroblastic); code 9533 (psammomatous); code 9534 (angiomatous); and code 9537 (transitional/mixed). Atypical meningiomas (165) were defined by ICDO codes of 9535 (hemangioblastic/angioblastic) or 9536 (hemangiopericytic). Malignant meningiomas (771) were defined as having an ICDO code of 9530/3.

\section{Demographic Variables}

Demographic data for all patients in this data set were complete for gender, age at diagnosis, vital status, and location of the tumor. Less than $10 \%$ of records for variables such as race, surgery, radiation treatment, and hospital type, had missing or unknown information. Approximately $17 \%$ of records had missing or unknown information for number of new cancer cases seen per year. Data on tumor size were missing for $57 \%$ of cases. However, we were unable to ascertain whether these variables were truly missing or simply not reported by some of the hospitals.

Five groupings were selected to classify age at diagnosis: 0 to 44 years, 45 to 64 years, 65 to 74 years, 75 to 84 years, and 85 years and older. Because of limited numbers of cases receiving radiation treatment, this variable was defined as "yes" (those patient receiving any form of radiation therapy) or "no" (those patients who did not receive radiation treatment). Tumor size was grouped into small $(<25 \mathrm{~mm}$ at the largest dimension), medium (25-49 $\mathrm{mm})$, and large $(50-100 \mathrm{~mm})$. Tumors recorded as being larger than $100 \mathrm{~mm}$ could not be verified and were not included in the analyses. The number of cancer cases seen in the hospital was grouped into less than 500 new cases per year, 500 to 799 new cases per year, and 800 or more new cases per year. Hospital type was divided into academic (National Cancer Institute Comprehensive Cancer Programs and Teaching Hospital Cancer Programs) and community (Community Hospital Comprehensive Cancer Programs and Community Hospital Cancer Programs).

\section{Statistical Analysis}

The data were converted from an ASCII file to a Statistical Analysis System (SAS) data set and SAS, Version 6.03 was used to provide frequencies, means, and survival estimates.[17] Chi-square estimates were calculated using EpiInfo, Version 6.03.[2] Benign, atypical, and malignant meningiomas were analyzed separately. Because of the small number of "events" in the atypical grouping $(<10$ in many instances), only primary analyses were performed. The Kaplan-Meier product-limit method[7] was used to determine survival rates for univariate models and comparisons were made using the log-rank test.[17] Observed survival rates were estimated and were not adjusted for deaths due to other causes. Study endpoints for survival models were death or date of last known contact. Information on recurrence was not complete for the years 1985 to 1986, and cases diagnosed during these years were excluded from these analyses. Recurrence that was recorded less than 12 months after diagnosis was excluded from analysis to minimize the potential for misclassification. The rate for recurrence of symptoms was defined as the time from tumor diagnosis to recurrence of clinical symptoms, regardless of the method of treatment. The rate for recurrence of the tumor was defined as the time from tumor diagnosis to recurrence of clinical symptoms after complete tumor removal. Endpoints for recurrence models were date of recurrence or date of last known contact. Individual factors that were statistically significant ( $\mathrm{p}<$ 0.05 ) were then entered simultaneously into a proportional hazards model, and significant predictors of survival and recurrence rates were selected using the stepwise approach.[17]

Functional capacity analyses were performed using logistic regression.[17] Individuals who were deceased were eliminated from the analyses. Endpoints for functional capacity analyses were: good, normal or symptomatic, but not requiring assistance; or poor, ambulatory greater than $50 \%$ or less than 
$50 \%$ of the time or bedridden, at the time of last known contact.

Preliminary univariate analyses allowed some values of certain variables to be collapsed. Mortality curves for Caucasian Hispanics were not significantly different from those for Caucasians. Similarly, the mortality curves for African American Hispanics were not significantly different from those for African Americans. Therefore, Caucasian Hispanics were grouped with Caucasians and African American Hispanics were grouped with African Americans. Patients with tumors of the calvaria and those with overlapping tumors were found to have similar survival rates, and these two groups were combined for further analyses. Any type of surgery was classified as "yes:" 5740 patients with partial, 1077 with total, and 1047 patients with radical resection. Under "no" were classified 1507 patients who did not undergo surgery, and 171 patients who had exploratory surgery or biopsy only. Unless otherwise noted, analyses for the current study were performed using these collapsed variables.

\section{RESULTS}

\section{Demographic Characteristics}

The median length of follow-up study was 10 months (range 0-93 months) for benign meningiomas, 12 months (range 0-79 months) for atypical meningiomas, and 12 months (range 0-90 months) for malignant meningiomas. Fifty-four percent of patients with benign meningioma were followed for less than 1 year; $44 \%$ of these were followed for less than 1 month, and $6.7 \%$ died within 1 month of diagnosis. Forty-eight percent of patients with atypical meningiomas were followed for less than 1 year; $44.3 \%$ of these were followed for less than 1 month, and 5.1\% were dead within 1 month postdiagnosis. Forty-seven percent of patients with malignant meningioma were followed for less than 1 year; $17.2 \%$ of these were followed for less than 1 month and $7.1 \%$ died within the 1st month of diagnosis. In contrast, $11.2 \%$ of benign and $10.9 \%$ of atypical compared with $14.1 \%$ of malignant meningioma patients were followed for at least 5 years.

The average age (61.3 years) at diagnosis for those with benign meningiomas was significantly older than the average age at diagnosis for those with atypical $(55.4$ years, $\mathrm{p}<0.001)$ or malignant $(59.7$ years, $\mathrm{p}<0.001)$ meningiomas; however, these differences are not likely to be clinically significant. A greater percentage of younger patients were observed to have malignant compared with benign meningiomas, although this difference was not significant (Table 1). A higher frequency of males had atypical or malignant meningiomas than benign meningiomas ( $p<0.0001$ for both, Table 1). However, more females than males developed all three histological types. No racial differences were noted between patients in the three groupings of meningiomas. 


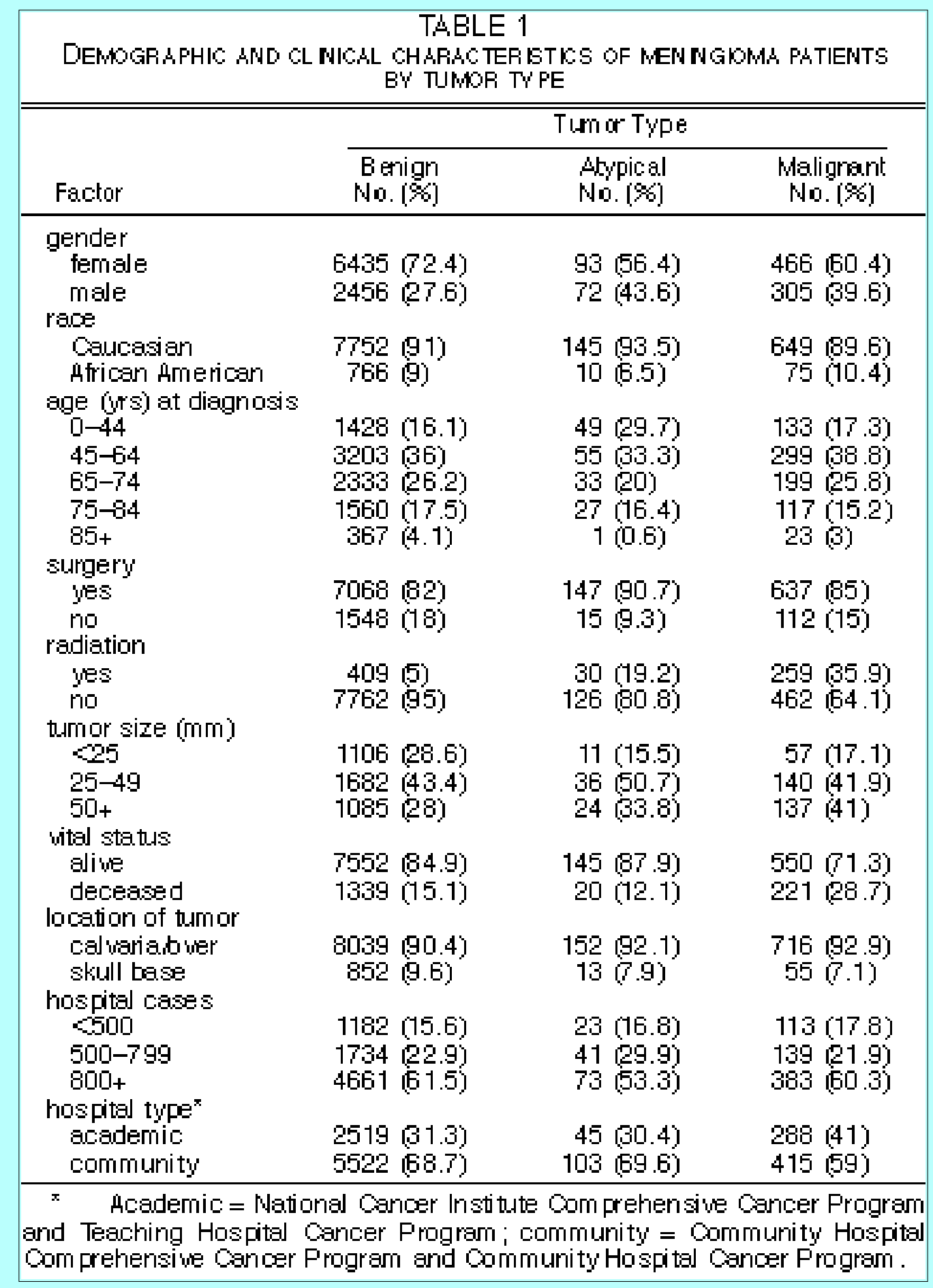

Noticeable differences in clinical factors between those patients with benign, atypical, and malignant meningiomas were observed (Table 1). Twenty-nine percent of patients with malignant meningiomas died compared with only $15 \%$ of those with benign and $12 \%$ of those with atypical meningiomas ( $\mathrm{p}<$ 0.0001 for both). Patients with malignant meningiomas were more likely to have undergone surgery $(\mathrm{p}=$ 0.04 ), to have received radiation therapy ( $<<0.0001)$, to harbor larger tumors ( $p<0.0001$ ), and to have overlapping tumors $(\mathrm{p}<0.0001)$ compared to those with benign meningiomas. In addition, patients with malignant meningiomas were seen less often at Community Hospital and Community Hospital Comprehensive Cancer Programs than those with benign tumors $(\mathrm{p}<0.0001)$. Patients with atypical meningiomas were also more likely to have undergone surgery $(\mathrm{p}=0.0004)$ and radiation therapy $(\mathrm{p}<$ $0.0001)$ than those with benign tumors, although tumor size and location did not differ. 


\section{Survival Rates}

The overall 2- and 5-year survival estimates for patients with meningioma, regardless of the tumor behavior, were $81 \%$ and $69 \%$, respectively. However, survival estimates were significantly different when stratified by histological type. Two-year survival estimates were $82 \%$ for those with benign meningiomas, $89 \%$ for those with atypical meningiomas, and $72 \%$ for those with malignant meningiomas. Those patients with benign meningiomas had a 5-year survival estimate of $70 \%$, compared with 5-year survival rates of $75 \%$ in those with atypical meningioma and $55 \%$ in those with malignant meningioma.

Age at diagnosis was a significant predictor of survival for patients with benign, atypical, and malignant meningiomas (Table 2). For all three groupings, increased survival was inversely related to age at diagnosis, with the youngest patients having the best survival rates. In patients with benign meningiomas, female gender, Caucasian race, surgery classified as yes, small tumor size, radiation treatment classified as no, location of the tumor in the skull base, and community hospitals were factors independently associated with increased survival rates (Table 2). In addition to age at diagnosis, increased survival rates for those patients with malignant meningiomas were predicted separately by female gender, surgery classified as yes, radiation treatment classified as no, and more than 800 new cancer cases seen per year by the treating hospital (Table 2). No additional variables were significant predictors of survival in those patients with atypical meningiomas. 


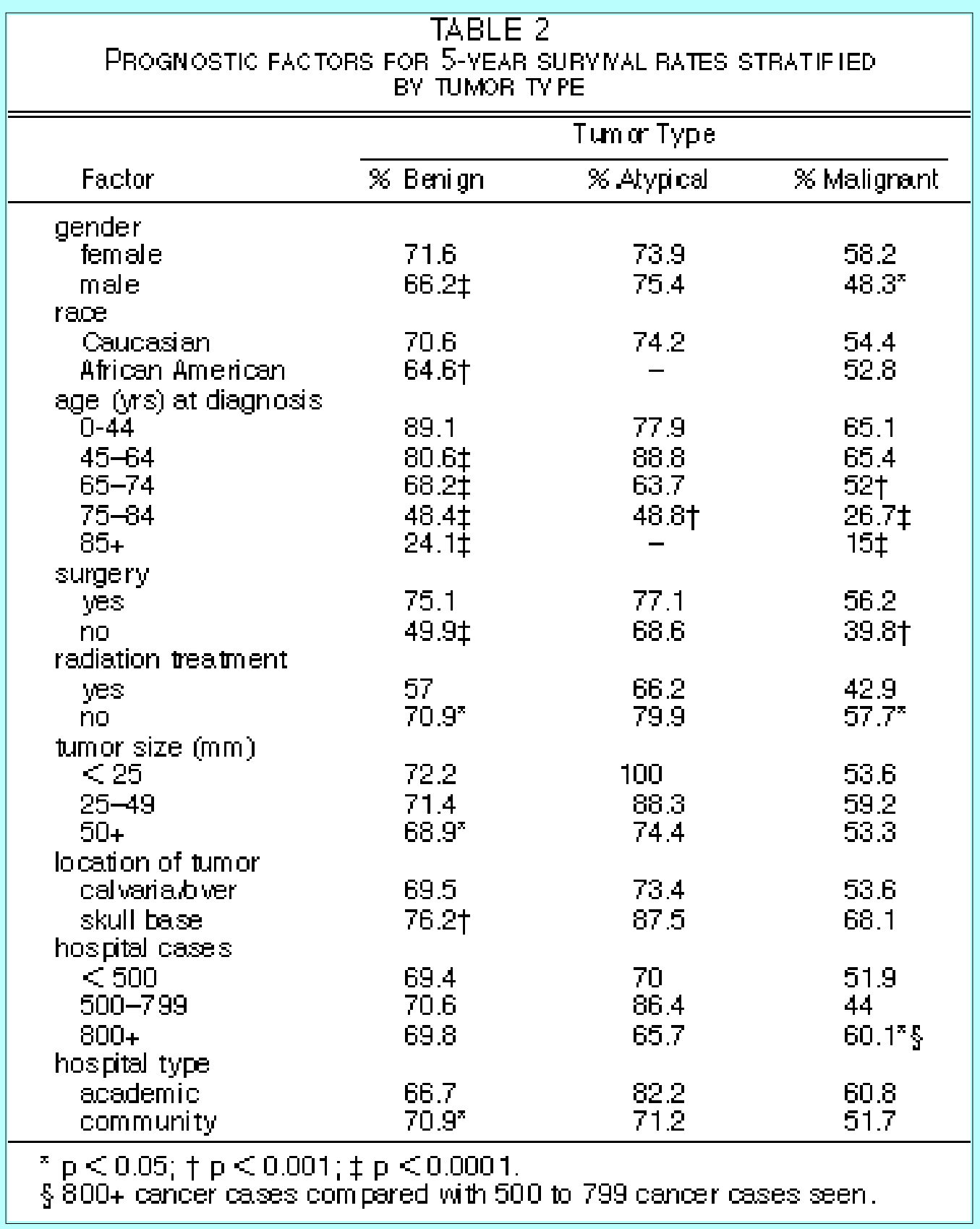

A crossover effect was noted for radiation therapy in both benign and malignant meningiomas, such that those patients who had received radiation treatment appeared to have better survival rates in the first 12 months after diagnosis than those who did not. However, for those patients who survived the first 12 months after diagnosis, those who had received radiation treatment had subsequent decreased survival compared with those who had not received radiation treatment. To separate this contrasting effect, radiation was separated into two time-dependent variables for the multivariate analyses. 


\section{TABLE 3}

MULTIYARIE ANALYSS OF SIGNIFKANT PREDICTORS OF MORTALTY IN PATIENTS WITH BEN GN MEN NGIOMAS*

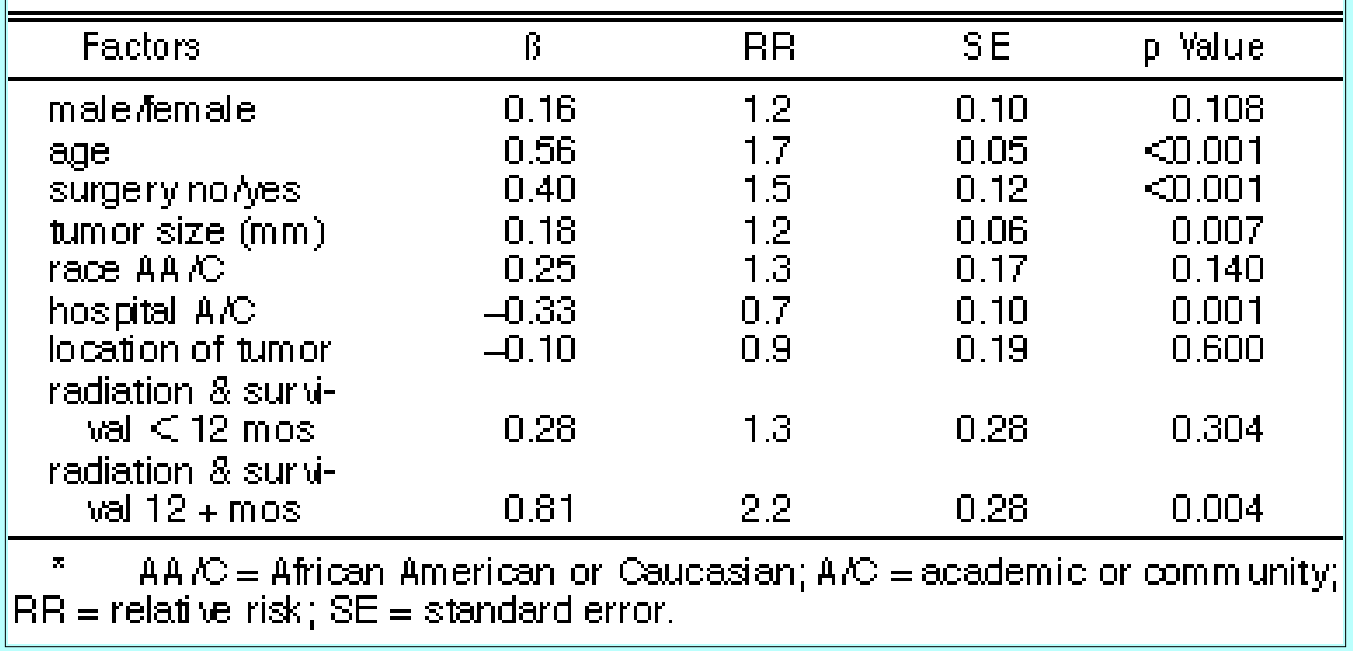

All variables significant at the univariate level were entered into a multivariate model to determine significant prognostic factors. Factors that remained significant in predicting survival for patients with benign meningiomas included age at diagnosis, surgery, tumor size, type of hospital, and radiation treatment in those who had survived at least 12 months (Table 3). After controlling for other factors, gender and race were not significant predictors of survival time. Figure 1 presents the estimated survival curves by age group at diagnosis after adjusting for radiation (yes/no), surgery (yes/no), hospital type, and tumor size.

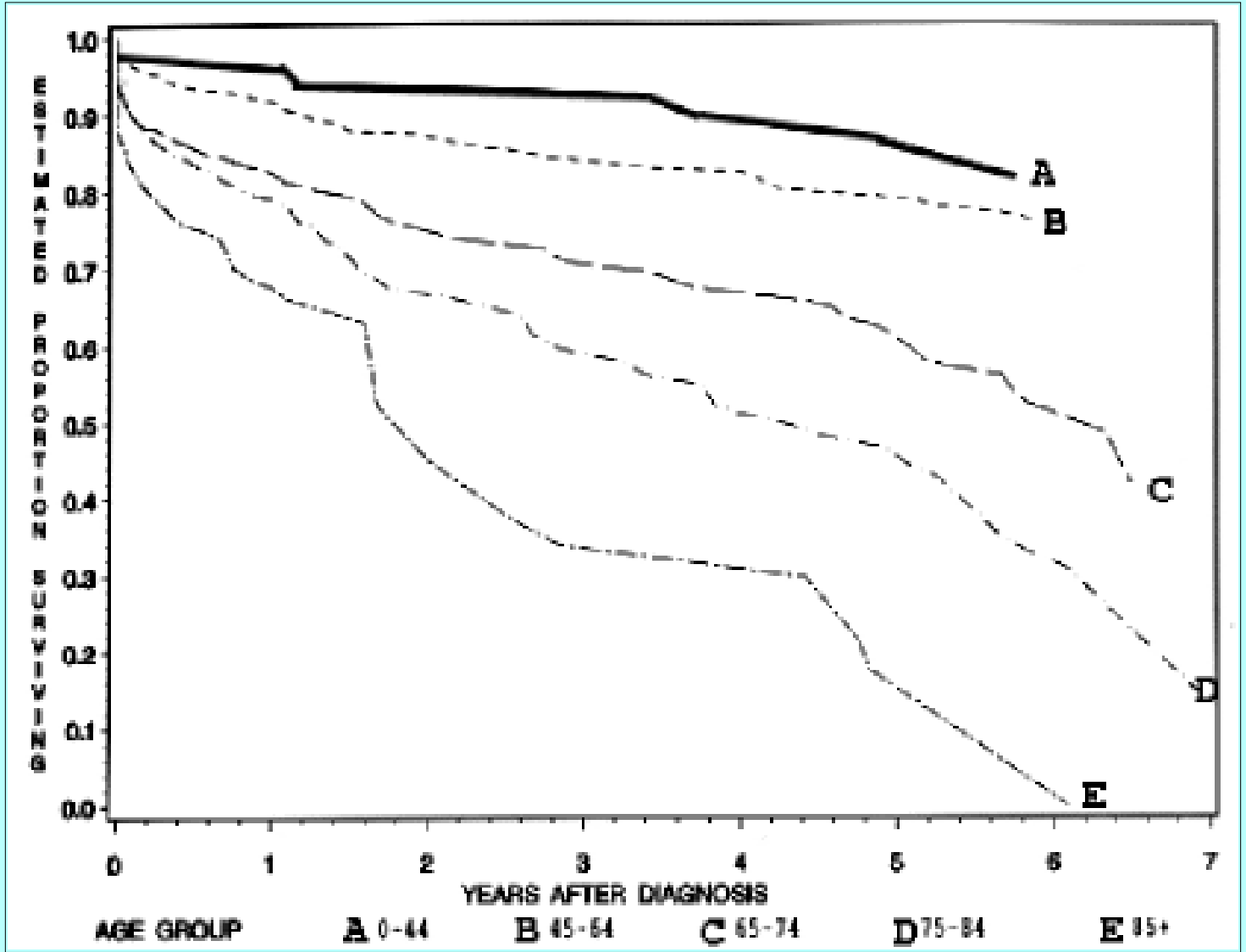

Fig. 1. Survival estimates by age group for patients with benign meningioma, adjusted for radiation $(\mathrm{Y} / \mathrm{N})$, surgery $(\mathrm{Y} / \mathrm{N})$, hospital type, and tumor size. 
Age at diagnosis, surgery, and no radiation treatment in those patients who had survived at least 12 months remained significant predictors of survival time for those with malignant meningiomas in the multivariate models (Table 4).

\begin{tabular}{|lcccc|}
\hline \multicolumn{5}{c|}{ TABLE 4 } \\
MULTIYAR LTE ANALYSS OF SIGNIFCANT PREDICTORS OF MORTALTY IN PATIENTS \\
WITH MLL GNANT MEN NGLMAS*
\end{tabular}

Similar to the results for benign meningiomas, gender was not a predictor of mortality after controlling for other factors. Estimated survival curves by age group at diagnosis after adjusting for radiation treatment (yes/no) and surgery (yes/no) are shown in Fig. 2. The survival curves for those patients with malignant meningiomas fall more rapidly in the early years after diagnosis compared with those harboring benign meningiomas.

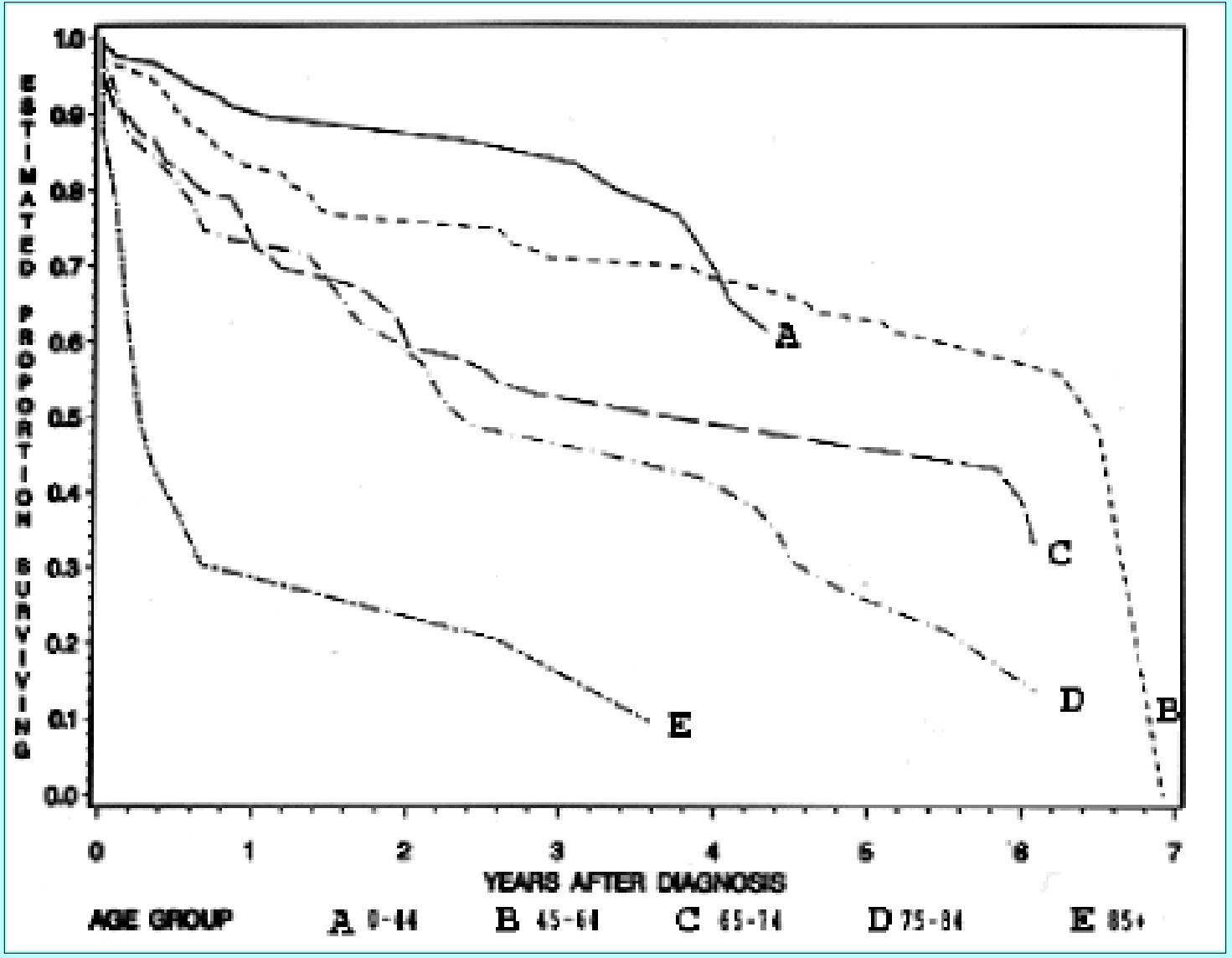

Fig. 2. Survival estimates by age group for patients with malignant meningioma, adjusted for radiation $(\mathrm{Y} / \mathrm{N})$ and surgery $(\mathrm{Y} / \mathrm{N})$. 


\section{Evaluation of Surgery and Radiation Therapy}

Cases of benign, atypical, and malignant meningioma were separated into four surgery/radiation therapy combinations: 1) no surgery, no radiation therapy; 2) radiation therapy only; 3) surgery only; and 4) both surgery and radiation therapy (Table 5). The greatest number of patients underwent surgery as the only treatment for benign, atypical, and malignant meningiomas. The fewest number of patients received radiation therapy alone.

\begin{tabular}{|c|c|c|c|c|c|c|}
\hline \multicolumn{7}{|c|}{$\begin{array}{l}\text { TABLE } 5 \\
\text { FIVEVEAR SURYYAL ESTMATES FOR PATIENTS WITH BEN GN, ATYPCAL, AND MAL GNANT MEN NGOMA } \\
\text { STRATFED BV SURGERY AND RADLTION THERAPY GROUP }\end{array}$} \\
\hline \multirow[b]{3}{*}{ Group } & \multicolumn{6}{|c|}{$\begin{array}{ll}\text { Timor Type } \\
\end{array}$} \\
\hline & \multicolumn{2}{|c|}{ B enign } & \multicolumn{2}{|c|}{ Alypical } & \multicolumn{2}{|c|}{ Malignant } \\
\hline & $\begin{array}{l}\text { No. of } \\
\text { Patients }\end{array}$ & $\begin{array}{l}\text { 5-Yr Su- } \\
\text { vival (\%) }\end{array}$ & $\begin{array}{l}\text { No. of } \\
\text { Patients }\end{array}$ & $\begin{array}{l}\text { 5-Yr Sur- } \\
\text { vival (\%) }\end{array}$ & $\begin{array}{l}\text { No. of } \\
\text { Patients }\end{array}$ & $\begin{array}{l}5-\text { Yr Sur } \\
\text { vival (\%) }\end{array}$ \\
\hline no surger yho radiation & 1340 & 49.9 & 10 & - & 77 & 36.7 \\
\hline radiation only & 108 & 25.1 & 3 & - & 31 & 36.9 \\
\hline surgery only & 6375 & 75.8 & 114 & 79.7 & 380 & 62.1 \\
\hline partial removal & 4577 & 75.3 & 86 & 88.0 & 279 & 63.8 \\
\hline total removal & 1798 & 76.9 & 28 & 65.4 & 101 & 57.1 \\
\hline surgeryładiation & 289 & 64.0 & 27 & 64.6 & 223 & 43.1 \\
\hline partial removal & 238 & 65.3 & 20 & 49.7 & 169 & 44.7 \\
\hline total removal & 51 & 57.8 & 7 & - & 54 & 40.8 \\
\hline
\end{tabular}

For patients with benign and malignant meningiomas, survival rates were not significantly different in those receiving radiation therapy only compared with those not undergong surgery or receiving radiation therapy ( $p>0.05$ ). For benign meningiomas, the two groups undergoing surgery had significantly better survival rates than the two groups that did not undergo surgery ( $p<0.0001$ for both). However, those receiving radiation therapy in addition to surgery, although initially appearing to do better, had worse survival rates in the long term than those who underwent surgery but did not receive radiation therapy $(\mathrm{p}$ $=0.09$; Fig. 3). For patients with malignant meningiomas, those undergoing surgery but not receiving radiation therapy did significantly better than all other groups $(\mathrm{p}<0.01)$. No differences in survival rates for those with atypical meningiomas were noted, primarily because of small numbers in the no surgery groups. 


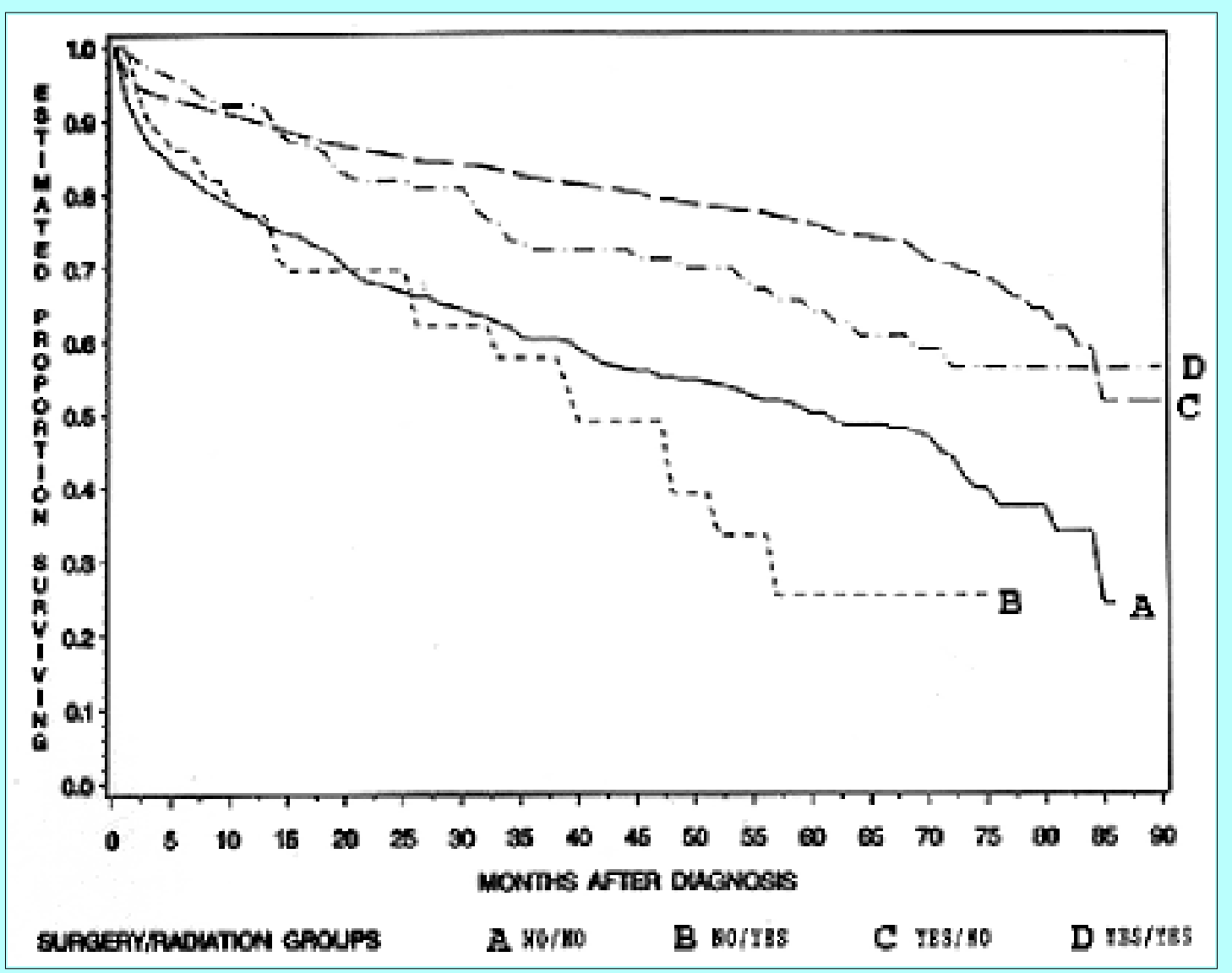

Fig. 3. Survival estimates by treatment group for patients with benign meningiomas who underwent surgery and/or received radiation therapy.

In those patients with benign meningiomas, there were no significant differences in the survival curves for those who underwent partial resections with or without radiation therapy or those in whom a total resection was accomplished with or without radiation therapy (Table 5). No significant differences were noted for those patients with malignant meningiomas who underwent total resections with or without radiation therapy, although those in whom a partial resection was achieved had significantly worse survival rates if they also received radiation therapy $(\mathrm{p}=0.02)$. Furthermore, in most instances, patients who underwent total removal of the tumor had worse 5-year survival rates than those having partial tumor removal.

\section{Recurrence Rates}

Valid information regarding recurrence status was obtained in 595 of the 8891 patients with benign meningioma, and 52 of those experienced a recurrence of symptoms. Valid recurrence status data were obtained in 56 patients with malignant meningioma, and nine of these had a recurrence of symptoms. The average time from tumor diagnosis to recurrence of symptoms was 31.2 months (range 12-139 months) for benign and 31.7 months (range 12-83 months) for malignant meningiomas. No analyses were performed on the data obtained in patients with atypical meningiomas because of extremely small sample size.

In the overall group, regardless of the method of treatment, the 5-year rate for recurrence of symptoms was $18.2 \%$ for those patients with benign tumors and $27.5 \%$ for those with malignant tumors. Analyses revealed that race $(\mathrm{p}=0.004)$, tumor site (overlapping vs. calvaria/skull base; $p<0.0001$ ), and tumor size $(p=0.02)$ were significant predictors of recurrence of symptoms for patients with benign 
meningiomas. Multivariate analysis of these statistically significant variables revealed that differences in race were accounted for by the other variables: tumor size $(R R=2.3, p=0.02)$ and site $(R R=9.2, p=$ 0.008 ) remained in the multivariate model. There were no statistically significant prognostic factors for recurrence of symptoms in patients with malignant meningiomas.

For those 176 patients with benign meningiomas in whom total or radical removal of the tumor was achieved, the 2-year and 5-year symptom recurrence rates were $4.7 \%$ and $20.5 \%$, respectively. Factors independently associated with recurrence were Caucasian race $(\mathrm{p}=0.006)$ and total tumor removal (compared with radical removal; $\mathrm{p}=0.0008$ ). Both variables remained significant upon entering them into a multivariate model $(R R=3.6, p=0.02$ for race and $R R=5.5, p=0.003$ for extent of resection).

\section{Functional Capacity}

Of those patients with benign tumors, valid functional capacity information was obtained in 4189 . Three thousand, eight hundred and twenty-four $(91.3 \%)$ were categorized as having good functional capacity (requiring no assistance), whereas $365(8.7 \%)$ required at least some assistance and were regarded as having poor functional capacity. For those patients with malignant meningiomas, 289 had valid functional capacity codes, $248(85.8 \%)$ of whom had good functional capacity and 41 (14.2\%) of whom had poor functional capacity.

Logistic regression analysis revealed that increasing age, no surgery, and a smaller number of cancer cases seen at the reporting hospital were all univariately associated with poor functional capacity in those patients with benign tumors ( $\mathrm{p}<0.005$ for all). All three variables remained in the multivariate model ( $\mathrm{p}$ $<0.001$ for all). The only variable predictive of functional capacity for those patients with malignant meningiomas was the number of cancer cases seen at the reporting hospital, with significantly more patients treated at hospitals seeing more than 800 new cancer cases per year reporting good functional capacity $(\mathrm{p}<0.007)$.

\section{DISCUSSION}

Clinically based studies usually restrict data collection to those patients who have received a certain type of treatment (such as surgery). This database, although not population based, consists of information collected from all newly diagnosed tumor patients in a voluntarily participating hospital regardless of their method of treatment, and thus, more closely reflects clinical and survival patterns of the population than hospital-based series. Therefore, prognostic factors related to survival rates can be assessed in the overall meningioma patient population.

In the current study, average follow-up study was longer for patients with malignant than for benign meningiomas, because more patients with benign meningioma were followed for less than 1 year. Reasons for the relative lack of follow up in this benign meningioma population are unknown, but may be related to a better posttreatment prognosis than for malignant meningiomas. This lack of follow up may bias the results such that survival and recurrence are underestimated if those individuals who have been lost to follow-up review are different from those who have been actively followed. In view of this, participating hospitals should be encouraged to engage in active follow-up evaluation of patients with meningioma and other brain tumors, particularly those with tumors of a benign nature. As the NCDB matures and the follow-up interval increases, the pattern of survival over a longer time period will stabilize and the probability of bias will decrease.

Age is a significant predictor of mortality in patients with benign, atypical, and malignant meningiomas. 
As age at diagnosis increases, dramatic decreases in the survival rate are apparent. These survival rates are based on observed values and do not take into account the underlying cause of death. Observed survival rates usually underestimate the true survival rate, especially in elderly patients who more often die from other causes. However, in many instances, the relative survival rate in patients with malignant brain tumors is similar to the observed survival rate.[1]

Gender and race, although individual prognostic factors for benign and malignant meningiomas, were explained by other factors in the final multivariate models. How and where patients are treated may explain some of the discrepancies found in the survival rates between races and gender. For benign meningiomas, the type of hospital in which a patient is treated may influence the survival rate. For patients with malignant meningiomas, however, hospital type and size do not appear in the final multivariate model.

The survival rate in patients with benign and malignant meningiomas is increased in those who have undergone surgery compared with those who have not received surgical treatment. Those patients undergoing surgery as the only method of treatment have the best 5-year survival rates. Conversely, those receiving radiation therapy as the only mode of treatment have the worst survival rates. The survival rate is also worse in those with total compared with partial surgical removal of the tumor. It is likely that patients who underwent the more radical surgical procedures and received radiation therapy had a worse prognosis at the time of diagnosis, as defined by clinical factors not available in this data set, and consequently, also had a worse survival rate. A selection bias probably exists in that patients with unresectable or otherwise dangerous meningiomas were preferentially referred for radiation therapy; however, an association between the type of therapy received and the expected outcome may exist in that radical therapy is used more frequently in patients with a better prognosis than in patients with advanced disease.[16] Certain patient characteristics that could determine the course of treatment, such as general health of the patient at diagnosis, extent of disease, and reason for no cancer-directed surgery, were not assessed in this data set and these questions could not be addressed.

National Cancer Database survival rates are lower than population-based survival rates for meningiomas (Table 6). The survival rates in the current study are dependent on voluntary reporting by hospitals and may be impacted by the types of hospitals submitting data. Misclassification of tumors could also account for a lower survival rate among NCDB cases. National Cancer Database survival rates are also lower than clinically based survival rates. Clinical studies generally only include patients who have had surgery to remove all or part of the tumor. Conversely, the NCDB includes those patients with meningiomas who did not undergo surgical or other active treatment, which reduces the survival estimates. 


\begin{tabular}{|c|c|c|c|c|}
\hline \multicolumn{5}{|c|}{$\begin{array}{c}\text { TABLE } 6 \\
\text { FIVE-YEAR SURYMAL ESTMTES FOR NCDB, POPULATION-BASED, } \\
\text { AND CL NICAL-BASED STUDIES } \\
\end{array}$} \\
\hline \multirow[b]{2}{*}{ Reference Study } & \multicolumn{4}{|c|}{ Tum or Type } \\
\hline & \%.Al & \% Benign & \% Alypical & \% Malignant \\
\hline NCDB & $\begin{array}{l}69 \\
64 \mathrm{M} \\
70 \mathrm{~F}\end{array}$ & 70 & 75 & 55 \\
\hline $\begin{array}{l}\text { population-based studies } \\
\text { Percy, et al., } 1972 \\
\text { Sant, et al., } 1988 \\
\text { Mahaley, et al., } 1989 \\
\text { Helseth \& Mork, } 1989 \\
\text { Sankila, et al., 1992 } \\
\text { Preston-Martin, et al., } 1993\end{array}$ & $\begin{array}{l}59 \\
83 \\
91 \\
84 \times \\
73 \\
87 \mathrm{M} \\
94 \mathrm{~F}\end{array}$ & & & 61 \\
\hline $\begin{array}{l}\text { clinical-based studies } \\
\text { Mirimanoff, et al., } 1985 \\
\text { Kallio, et al., } 1992 \\
\text { Mahm ood, et al., } 1993 \\
\text { Goldsmith, et al., } 1994 \\
\text { Younis, et al., } 1995\end{array}$ & 83 & $\begin{array}{l}83 \\
87 \\
85\end{array}$ & 58 & $\begin{array}{l}81 \\
60 \\
58 \\
62\end{array}$ \\
\hline
\end{tabular}

Although date of first treatment was recorded in this data set, the order of treatment was not obtained. Therefore, recurrence risks were calculated from the time of diagnosis. In this data set $95.3 \%$ of benign and $96.3 \%$ of malignant meningioma patients received treatment in the same year in which they were diagnosed. The 5-year tumor recurrence rate for benign meningiomas in those undergoing complete tumor removal was $20.5 \%$ in the current study. This rate is higher than that found in the clinical literature (Table 7). Mirimanoff, et al.,[12] found that the recurrence rate for benign meningiomas was $7 \%$ at 5 years, and only $20 \%$ after 10 years posttreatment. The recurrence rate for benign meningiomas in a study by Mahmood, et al.,[9] was $2 \%$ at 5 years. The elevated rate of recurrence in the current study may be due to imprecise definitions of recurrence used in the data base and a more inclusive data base.

\begin{tabular}{|c|c|c|c|}
\hline \multicolumn{4}{|c|}{$\begin{array}{c}\text { TABLE } 7 \\
\text { FIVEVEAR RECURRENCE AND PROGRESSION RATES FOR PATIENTS WITH } \\
\text { MEN NGOMAS WHO WERE TREATED BY COMPLETE OR PARTIAL RESECTION }\end{array}$} \\
\hline \multirow[b]{2}{*}{ Reference Study } & \multicolumn{3}{|c|}{ Termor Type } \\
\hline & \% Benign & \%.Alypical & \% Malignant \\
\hline \multicolumn{4}{|l|}{ complete resection } \\
\hline Mirimanoff, et al., 1985 & 7 & & \\
\hline Jääskeläinen, et al , 1986 & 3 & 38 & \\
\hline $\begin{array}{l}\text { Mahm ood, et al., } 1993 \\
\text { Younis et al. } 1995\end{array}$ & 2 & 50 & 33 \\
\hline \multicolumn{4}{|l|}{ partial resection } \\
\hline Mirimanoff, et al., 1985 & 37 & & \\
\hline Mahmood, et al., 1993 & & 50 & 100 \\
\hline $\begin{array}{l}\text { Goldsmith, et al., } 1994 \\
\text { Younis, et al., } 1995\end{array}$ & 11 & & $\begin{array}{c}52 \\
100^{x}\end{array}$ \\
\hline
\end{tabular}


A limitation of the study was the inability of the authors to validate and control the quality of data. For some variables, a small percentage of the data were missing; race, surgery, radiation treatment, and hospital type each had less than $10 \%$ of cases with missing information (Table 1). However, a few variables, such as tumor size and number of new cancer cases seen, had a large number of records with missing or unknown information (57\% and $17 \%$, respectively). These records with missing or unknown codes had to be excluded from the analyses. The possibility of bias exists, but the exclusion of these data may have been systematic. For example, any one institution may not have submitted information on a particular variable for any of its patients, regardless of clinical presentation or outcome. However, we were unable to determine the percentage of records that contained truly missing information as compared with those that were simply not reported by the participating hospital.

Information on diagnostic confirmation of the tumors was not available, and the possibility exists that misclassification or misdiagnosis may have occurred. The classification of meningiomas was based on ICDO codes recorded in the medical records; meningiomas with unusual cytological features may have been incorrectly classified as benign. Because of the limited information available regarding the pathological characteristics of the tumors, we were unable to determine if misclassification occurred. In addition, no information on other clinical factors that may be important in the prognosis of survival time, such as extent of disease, clinical presentation, or coexisting conditions, were collected for this registry, and thus, were not controlled for in multivariate analyses. It would be difficult to assess what effect these factors may have on the results presented here.

More detail on clinical variables in a broader patient population would enable better estimates of survival while controlling for pretreatment prognosis. Participation in data collection for NCDB will be required for all hospitals accredited by the Commission of Cancer in 1996. This will provide a larger database of newly diagnosed benign and malignant cancers from across the United States. As data quality and follow-up time increase, this data set will become invaluable for clinical and epidemiological studies.

\section{Acknowledgments}

The authors would like to thank the NCDB Data Request Committee and Mr. Herman Menck at the American College of Surgeons for providing the data.

\section{References}

1. CBTRUS: 1996 Annual Report. Chicago: Central Brain Tumor Registry of the United States, 1997

2. Dean AG, Dean JA, Coulombier D, et al: Epi Info, Version 6. Revised for Version 6.03. Atlanta: Centers for Disease Control and Prevention, 1995

3. Goldsmith BJ, Wara WM, Wilson CB, et al: Postoperative irradiation for subtotally resected meningiomas. A retrospective analysis of 140 patients treated from 1967 to 1990 . J Neurosurg 80:195-201, 1994

4. Helseth A, Mørk SJ: Primary intraspinal neoplasms in Norway, 1955 to 1986. A population-based survey of 467 patients. J Neurosurg 71:842-845, 1989

5. Jääskeläinen J, Haltia M, Servo A: Atypical and anaplastic meningiomas: radiology, surgery, radiotherapy, and outcome. Surg Neurol 25:233-242, 1986 
6. Kallio M, Sankila R, Hakulinen T, et al: Factors affecting operative and excess long-term mortality in 935 patients with intracranial meningioma. Neurosurgery 31:2-12, 1992

7. Kaplan EL, Meier P: Nonparametric estimation from incomplete observations. J Am Stat Assoc 53:457-481, 1985

8. Mahaley MS Jr, Mettlin C, Natarajan N, et al: National survey of patterns of care for brain-tumor patients. J Neurosurg 71:826-836, 1989

9. Mahmood A, Caccamo DV, Tomecek FJ, et al: Atypical and malignant meningiomas: a clinicopathological review. Neurosurgery 33:955-963, 1993

10. Menck HR: A preliminary study of National Cancer Data Base representativeness, in Steele GD Jr, Winchester DP, Menck HR, et al (eds): National Cancer Data Base Annual Review of Patient Care 1993. Atlanta: American Cancer Society, 1993, pp 93-102

11. Menck HR, Garfinkel L, Dodd GD: Preliminary report of the National Cancer Data Base. CA Cancer J Clin 41:7-18, 1991

12. Mirimanoff RO, Dosoretz DE, Linggood RM, et al: Meningioma: analysis of recurrence and progression following neurosurgical resection. J Neurosurg 62:18-24, 1985

13. Percy AK, Elveback LR, Okazaki H, et al: Neoplasms of the nervous system. Epidemiologic considerations. Neurology 22:40-48, 1972

14. Preston-Martin S, Staples M, Farrugia H, et al: Primary tumors of the brain, cranial nerves and cranial meninges in Victoria, Australia, 1982-1990: patterns of incidence and survival.

Neuroepidemiology 12:270-279, 1993

15. Sankila R, Kallio M, Jääskeläinen J, et al: Long-term survival of 1986 patients with intracranial meningioma diagnosed from 1953 to 1984 in Finland. Comparison of the observed and expected survival rates in a population-based series [published erratum appears in Cancer 71:1394, 1993] Cancer 70:1568-1576, 1992

16. Sant M, Crosignani P, Bordo BM, et al: Incidence and survival of brain tumors: a population-based study. Tumori 74:243-252, 1988

17. SAS/STAT User's Guide, Version 6, ed 4. Cary, NC: SAS Institute, Inc., Vols 1 and 2, 1988

18. Smart CR, Menck HR, Eberle C, et al: An assessment of National Cancer Data Base quality control, in Steele GD Jr, Winchester DP, Menck HR, et al (eds): National Cancer Data Base Annual Review of Patient Care 1993. Atlanta: American Cancer Society, 1993, pp 103-111

19. Surawicz TS, Davis FG, Barlas S, et al: Brain tumor survival: results from the National Cancer Database. J Neurosurg 84:339A-340A, 1996 (Abstract)

20. World Health Organization: International Classification of Diseases for Oncology. Geneva: World Health Organization, 1990

21. Younis GA, Sawaya R, DeMonte F, et al: Aggressive meningeal tumors: review of a series. J Neurosurg 82:17-27, 1995 
Manuscript received February 17, 1997.

Accepted in final form March 17, 1997.

This work was conducted under contract to the Central Brain Tumor Registry of the United States supported by the Pediatric Brain Tumor Foundation of the United States through the Ride for Kids Fundraising Program sponsored by the American Honda Motor Company, Motorcycle Division.

Address reprint requests to: Bridget J. McCarthy, Ph.D., Department of Biostatistics/Epidemiology, University of Illinois at Chicago, 2121 West Taylor, Chicago, Illinois 60612. 